\title{
Impaired L-arginine metabolism marks endothelial dysfunction in CD73-deficient mice
}

\author{
P. Mierzejewska ${ }^{1}$ - M. A. Zabielska ${ }^{1,2} \cdot$ B. Kutryb-Zajac ${ }^{1} \cdot$ M. Tomczyk ${ }^{1} \cdot$ P. Koszalka ${ }^{3} \cdot$ R. T. Smolenski ${ }^{1}$. \\ E. M. Slominska ${ }^{1}$ (D)
}

Received: 17 October 2018 / Accepted: 12 April 2019 / Published online: 15 May 2019

(C) The Author(s) 2019

\begin{abstract}
Changes in the ecto-5'-nucleotidase activity - an extracellular nucleotide catabolic enzyme may lead to the inflammation and endothelial dysfunction. We investigated the effect of CD73 deletion on the endothelial function and L-arginine metabolism in various age groups of mice. 1-,3-,6-, and 12-month-old, male C57BL/6 J wild type (WT) and C57BL/6 J CD73-/- (CD73-/-) mice were used. Blood samples were used for the analysis of adenine nucleotide concentrations. Serum samples were analyzed for the concentration of amino acids, Interleukin 6 (IL-6), Intercellular Adhesion Molecule 1 (ICAM-1), Vascular Cell Adhesion Molecule 1 (VCAM-1), and endothelial nitric oxide synthase (eNOS) level. Serum and aortic nitrate/nitrite, as well as aortic arginase and NOS activity in endothelial cells (EC) were evaluated. CD73 deletion led to age-dependent increase in IL-6, ICAM-1, and VCAM-1 concentration compared to WT. All CD73-/- mice age groups were characterized by reduced L-Arginine concentration and eNOS level. Significantly lower NOS activity was noticed in EC isolated from CD73-/- mice lungs in comparison to EC isolated from WT lungs. The L-Arginine/ADMA ratio in the CD73-/- decreased in age-dependent manner in comparison to WT. The nitrate/nitrite ratio was reduced in serum and in aortas of 6-month-old CD73-/- mice as compared to WT. The ornithine/arginine and ornithine/citrulline ratios were increased in CD73-/- compared to controls. Blood (erythrocyte) Adenosine-5'-triphosphate and Adenosine-5'-diphosphate levels were reduced in favor to higher blood Adenosine-5'-monophosphate concentration in CD73-/- mice in comparison to WT. The CD73 deletion leads to the development of age-dependent endothelial dysfunction in mice, associated with impaired L-arginine metabolism. CD73 activity seems to protect endothelium.
\end{abstract}

Keywords Endothelium $\cdot$ Endothelial dysfunction $\cdot$ Nucleotide metabolism $\cdot$ Ecto-5'-nucleotidase $\cdot$ Adenosine $\cdot$ L-Arginine metabolism

Electronic supplementary material The online version of this article (https://doi.org/10.1007/s11010-019-03537-4) contains supplementary material, which is available to authorized users.

E. M. Slominska

eslom@gumed.edu.pl

1 Department of Biochemistry, Medical University of Gdansk, Debinki 1, 80-211 Gdańsk, Poland

2 Department of Physiology, Medical University of Gdansk, Gdańsk, Poland

3 Department of Medical Biotechnology, Intercollegiate Faculty of Biotechnology UG-MUG, Medical University of Gdansk, Gdańsk, Poland

\section{Introduction}

Nucleotides and their catabolites play a significant role in the cell energetics and regulation, where they act through both, intracellular and extracellular mechanisms. In the extracellular space, they are important regulators of the inflammation and immune response [1]. Adenine nucleotides and nucleosides are degraded by cell surface ecto-enzymes. One of the most important is ecto-5'-nucleotidase, also known as CD73. It catalyzes the hydrolysis of the adenosine-5'monophosphate (AMP) to adenosine [2]. CD73 has a wide tissue distribution. It is present in kidney, liver, lung, brain, and heart, as well as on leukocytes, thymus, spleen, and lymph nodes. Such widespread CD73 distribution is related to its high abundance in endothelium [3]. The adenosine, produced by $\mathrm{CD} 73$, is considered a molecule with an 
important signaling role, which is mediated by the activation of the P1 receptors. The decrease in the extracellular adenosine concentration may lead to the development of the inflammation and endothelial dysfunction [4].

Endothelial dysfunction is involved in the pathophysiology of all cardiovascular diseases. It is manifested by the increased permeability of endothelial cells, elevated adhesion molecules expression, intensified cytokines and chemokines secretion, leukocytes adhesion, platelet activation, and also facilitation of migration and proliferation of vascular smooth muscle cells [5]. The mechanisms underlying this disorder are related to an active inflammatory process and reduced bioavailability of nitric oxide (NO). $\mathrm{NO}$ is mainly formed in endothelial cells from L-arginine by endothelial NO synthase (eNOS) [6].

Our earlier studies demonstrated the strong association between the lack of the CD73 activity and the development of the aortic valve dysfunction in mice [7]. It is also known, that binding of the lymphocytes to the endothelium masks endothelial CD73 without any covalent modification [8]. Reduced level of extracellular adenosine on lymphocyte that contact with endothelial cells (EC) leads to an increased permeability of EC that contributes to enhanced leukocyte transmigration $[9,10]$. Other literature data also confirmed the involvement of CD73 in modulation of coronary vascular tone and platelet activation, as well as inhibition of leukocyte adhesion [11]. This study aimed to investigate the effect of the CD73 deletion on the endothelial function in mice at various age and relate it to L-arginine-dependent signaling pathways.

\section{Materials and methods}

\section{Animals maintenance}

All experiments were conducted in accordance with a Guide for the Care and Use of Laboratory Animals published by the European Parliament, Directive 2010/63/EU and were performed with approval of the Local Ethical Committee for Animal Experimentation in Bydgoszcz (27/2016). C57BL/6 J CD73-/- (CD73-/-) mice were obtained from Heinrich-Heine-Universität in Düsseldorf, Germany [11]. C57BL/6 J Wild Type (WT) $(n=27)$ and CD73-/- $(n=27)$ mice, fed standard chow diet, were used for the experiments. At the age of 1, 3, 6, and 12 months, mice were subjected to anesthesia-ketamine $(100 \mathrm{mg} / \mathrm{kg})$ and xylazine $(10 \mathrm{mg} /$ $\mathrm{kg}$ ). Blood and serum samples were collected and immediately frozen in liquid nitrogen. Blood samples were collected through the tail vein puncture of live mice. Following tail vein puncture, blood dripped directly into the Eppendorf tube previously cooled in liquid nitrogen, after which the bleeding was stopped with the gauze. Serum samples were obtained from peripheral blood after centrifugation $\left(\times 1700 \mathrm{~g}, 10 \mathrm{~min}, 2{ }^{\circ} \mathrm{C}\right)$. Then, after opening the chest, the thoracic and abdominal aortas were removed, placed in physiological saline and dissected from the surrounding tissues.

\section{Determination of amino acids concentration}

To evaluate the concentration of plasma amino acids, as well as L-arginine analogs, an aliquot of serum $(50 \mu \mathrm{l})$ was extracted with acetonitrile (ratio 1:2.4) and centrifuged $\left(\times 20,800 \mathrm{~g} / 10 \mathrm{~min} / 4{ }^{\circ} \mathrm{C}\right)$. Supernatant were collected and freeze dried. The precipitate was dissolved in water at a volume equal to the initial plasma volume. Amino acids and derivatives concentration were determined using high performance liquid chromatography-mass spectrometry (LC/ MS). The system contained a Surveyor MS autosampler, quaternary Surveyor MS pump, and a degasser connected to TSQ Vantage triple quadrupole mass detector. Heated electrospray ionization in positive mode was used. The column used for separation was a $50 \times 2 \mathrm{~mm}$ Synergi Hydro-RP 100 with a particle size of $2.5 \mu \mathrm{m}$. Mobile phase composed of water with $5 \mathrm{mM}$ nonafluoropentanoic acid (A) and acetonitrile with $0.1 \%$ formic acid (B). Mobile phase was delivered at $0.2 \mathrm{ml} / \mathrm{min}$ and volume of injection was $2 \mu \mathrm{l}$. The identity of individual amino acids and internal standard 2-chloroadenosine was confirmed by the similarity of molecular weights, fragmentation pattern, and chromatographic retention time [12].

Intracellular adhesion molecule-1 (ICAM-1), vascular adhesion molecule-1 (VCAM-1), interleukin 6 (IL-6), and tumor necrosis factor-alpha (TNF-alpha) concentration, as well as endothelial nitric oxide synthase level (eNOS) measurements.

The soluble ICAM-1, VCAM-1, and IL-6 serum levels (Sigma-Aldrich, RAB0220, RAB0506, RAB0308, RAB0477), as well as eNOS content (Wuhan EIAan Science, E0815 m) were determined using enzyme-linked immunosorbent assay kits according to the manufacturer's protocols.

\section{Isolation of murine lung endothelial cells}

For isolation, five CD73-/- and eight WT mice at the age of about 8 weeks were used for our procedure. Mice were anesthetized with ketamine $(140 \mathrm{mg} / \mathrm{kg})$ and xylazine ( $14 \mathrm{mg} / \mathrm{kg}$ ) followed by opening the chest. Murine lung tissues were harvested, placed into Petri dishes containing Dullbecco's Modified Eagle's Medium (DMEM) with low glucose supplemented with penicillin-streptomycin (SigmaAldrich), and cut into small pieces. The minced tissue and DMEM were collected in a $15 \mathrm{ml}$ tube and centrifuged (234 g, rt, $5 \mathrm{~min}$ ) to remove erythrocytes and blood plasma. 
Supernatant has been removed. Tissue pellet was washed with supplemented DMEM and centrifuged again (under the conditions mentioned above). Then, the tissue pellet was suspended in Collagenase Type A solution $(2.5 \mathrm{mg} / \mathrm{ml}$ in a DMEM low glucose, Gibco) mixed and incubated at $37{ }^{\circ} \mathrm{C}$ for $60 \mathrm{~min}$. After digestion with collagenase, the $70 \mu \mathrm{m}$ cell strainer was used to get the single cell suspension. The cell pellet was washed in Dulbecco's Phosphate-Buffered Saline (DPBS) and centrifuged twice (234 g, rt, $5 \mathrm{~min}$ ). After final centrifugation, a supernatant was removed and cells were resuspended in DMEM with D-Valine (glucose $4.5 \mathrm{~g}$ / 1 (Immuniq), 10\% FBS, Endothelial cell growth supplement-ECGS $15 \mathrm{mg} / 500 \mathrm{ml}, 2 \mathrm{mM}$ L-glutamine and penicillin-streptmycin) and plated into a T-25 tissue culture flask. The next day, the medium containing suspended and weakly adherent cells was transferred to a new culture flask. For the experiments cells between two to five passage were used.

\section{Measurement of nitric oxide synthase activity in murine endothelial cells}

For determination of endothelial nitric oxide synthase activity, CD73-/- and WT lung endothelial cells were cultured in 96 well plates at a density 100,000 cells per well. After $24 \mathrm{~h}$, nitric oxide production from murine EC was assessed using spectrofluorimeter Niric Oxide Synthase Detection System (Sigma-Aldrich, FCANOS1), according to the manufacturer's protocol. For fluorometric NO measurement, the cell-permeable NOS-derived NO-sensitive 4,5-diaminofluorescein diacetate (DAF-2DA) was used. Diphenyleneiodonium chloride (DPI) was used as an ihibitor of inducible NOS (iNOS) at a concentration of $1 \mathrm{uM}$ (as recommended in the protocol). The results are shown as relative fluorescence units (RFU) of NO concentration per mg of protein.

\section{Determination of serum and aortic nitrate/nitrite levels}

Serum nitrate/nitrite concentrations were determined using the nitrite/nitrate assay kit (Sigma-Aldrich, 23479) according to the manufacturer's protocol. NO metabolites concentration was measured by the Griess reaction, in which $\mathrm{NO}_{3}{ }^{-}$is converted to $\mathrm{NO}_{2}{ }^{-}$by the nitrate reductase. Following the enzymatic reaction, the Griess reagent was added to the samples. The absorbance of the azo compound was measured spectrophotometrically at a wavelength of $540 \mathrm{~nm}$. The azo coupling between diazonium species, which are produced from sulfanilamide with $\mathrm{NO}_{2}$, and naphthylethylenediamine resulted in a colorimetric product proportional to the NO metabolite present.

CD73-/- and WT mice aortic fragments for the nitrate/nitrite concentration measurement were homogenized in phosphate buffer ( $\mathrm{pH}$ 7.4) containing Tris- $\mathrm{HCl}$
(50 mM), EDTA (0.1 mM), phenylmethylsulphonyl fluoride $(0.1 \mathrm{mmol} / \mathrm{l})$, dithiothreitol $(0.5 \mathrm{mmol} / \mathrm{l})$, trypsin inhibitor $(10 \mu \mathrm{g} / \mathrm{ml})$, and leupeptin $(10 \mu \mathrm{g} / \mathrm{ml})$. The homogenates were centrifuged $\left(\times 20,000 \mathrm{~g}, 4^{\circ} \mathrm{C}, 15 \mathrm{~min}\right)$ and the supernatants were collected. The total nitrate/nitrite ratio was determined as mentioned above. NO metabolites levels are shown in $\mathrm{nmol} / \mathrm{mg}$ of tissue [13].

\section{Measurement of arginase activity}

To evaluate arginase activity in aortic fragments of CD73-/- and WT mice, the Arginase Activity Assay Kit (Sigma-Aldrich, MAK112) was used according to the manufacturer's protocol. Aortas were homogenized in $50 \mathrm{mM}$ Tris-HCl pH 9, centrifuged $\left(\times 5000 \mathrm{~g}, 4{ }^{\circ} \mathrm{C}, 5 \mathrm{~min}\right)$ and the supernatant was rebuffed using a $10 \mathrm{kDa}$ Molecular Weight Cut-Off Filter [14]. The arginase activity is shown as units per $g$ of tissue. One unit of arginase is the amount of enzyme that will convert $1 \mu \mathrm{mol}$ of $\mathrm{L}$-arginine to ornithine and urea per minute at $\mathrm{pH} 9.0$ in $37^{\circ} \mathrm{C}$.

\section{Determination of blood nucleotides and metabolites concentration}

To determine nucleotides and their metabolites concentration, the whole blood samples were immediately frozen in a liquid nitrogen, extracted with $1.3 \mathrm{M} \mathrm{HClO} 4$ (ratio 1:1) followed by a centrifugation $\left(\times 20,800 \mathrm{~g} / 15 \mathrm{~min} / 4{ }^{\circ} \mathrm{C}\right)$. Supernatants were then collected and brought to $\mathrm{pH} 6.0-6.5$ using $3 \mathrm{M} \mathrm{K3PO} 4$ solution. After 15-min incubation on ice, samples were centrifuged again $\left(\times 20,800 \mathrm{~g} / 15 \mathrm{~min} / 4{ }^{\circ} \mathrm{C}\right)$, and the supernatants were analyzed using high performance liquid chromatography (HPLC) as previously described [15]. Blood AMP hydrolysis rate was determined as described in the Supporting Informations.

\section{Statistical analysis}

The results were presented as mean \pm SEM. The statistical analysis was performed using Graph Pad Prism 7 (Graph Pad Software). Unpaired Student $t$ test was used for comparisons between two groups. Two-way analysis of variance (ANOVA) followed by Tukey's Multiple Comparison Test was used to compare more than two groups. A $p$ value $<$ 0.05 was considered a significant difference.

\section{Results}

To assess the development of inflammation and confirm the pro-inflammatory phenotype of the studied animals, the concentrations of ICAM-1, VCAM-1, and IL- 6 were measured in the 1-, 3-, 6-, and 12-month-old CD73-/- and WT 
mice serum. The level of both adhesion molecules, associated with the endothelium activation, as well as IL-6 were increased in CD73-/- mice serum as compared to WT, irresprecitive of their age (Fig. 1). The highest concentration of ICAM-1 was observed in 3- and 6-month-old mutants (Fig. 1a). The most increased IL-6 level was noticed in the 12-month-old-CD73-/- mice serum. Additionaly, the concentration of another pro-inflammatory factor-TNF-alpha was measured in the 6-month-old CD73-/- and WT mice serum. The level of this cytokine was approximately three times higher in mutant mice serum in comparison to control group (Supplementary Fig. S1).

The analysis of serum L-Arginine concentration, L-Arginine to asymmetric dimethyl arginine (ADMA) ratio and eNOS level, as well as NOS activity in the endothelial cells isolated from CD73-/- and WT mice are consistent with the development of endothelial dysfunction in mutant mice (Fig. 2). CD73-/- mice were characterized by significantly decreased L-Arginine concentration and considerably lower eNOS level in the serum, regardless of their age (Fig. 2a, c). Moreover, the decrease in the L-Arginine to ADMA ratio, which is strongly related to endothelial dysfunction, was proportional to the age of CD73-/- mice (Fig. 2b). Consistent with the lower eNOS level in serum, the activity of eNOS was significantly reduced in the endothelial cells isolated from CD73-/- mice as compared to WT (Fig. 2d).

We also investigated the L-Arginine analogs concentration: ADMA, L-NG-monomethyl arginine (L-NMMA) and symmetric dimethylarginine (SDMA) in the 1-, 3-, 6- and 12-month-old CD73-/- and WT mice serum. The agedependent ADMA increase in CD73-/- mice was noticed, as compared to WT (Fig. 3a). There were no significant differences between the L-NMMA and SDMA concentration in control and mutant mice serum, irrespective of their age (Fig. 3b, c).

To further characterize the implications of a lack of CD73 activity for the L-Arginine metabolism, we evaluated serum and aortic nitrate/nitrite levels, as well as arginase activity in the 6-month-old CD73-/- and WT mice aortas. The nitrate/nitrite ratio was markedly decreased in serum, but also in the aortic fragments of CD73-/- mice as compared to WT (Fig. 4a, b). In addition, we noticed considerably higher activity of arginase in the CD73-/- mice aortas in comparison with controls (Fig. 4c).

In the next step, we evaluated the L-Arginine metabolites concentration. We observed the significant increase in ornithine level in the CD73-/- in comparison to control, in particular in 1- and 3-month-old animals (Fig. 5a). There was no difference in citrulline concentration between study groups. Age did not affect changes in the L-Arginine metabolites in the serum of the mice involved in the experiment (CD73-/- and WT) (Fig. 5a, b). Moreover, we observed significantly increased ratios of ornithine/arginine and ornithine/citrulline in all CD73-/- age groups in comparison with controls (Fig. 5c, d).

Additionally, we measured the adenine nucleotides and metabolites concentration in whole blood samples of 6-month-old CD73-/- and WT mice. CD73-mutants were characterized by decreased blood ATP and ADP levels and-on the other hand-significantly higher blood concentration of AMP as compared to WT. The adenosine level was considerably lower in CD73-/- mice in comparison to WT (Fig. 6a), which was consistent with deletion of the CD73 encoding gene. The ATP/ADP ratio didn't differ between both study groups; however, ATP/ AMP ratio was significantly lower in CD73-deficient animals (Fig. 6b, c). Furthermore, we observed the significantly reduced AMP hydrolysis rate in mutant mice blood (Supplementary Fig. S2).

Moreover, we also analyzed the other amino acids concentration in 6-month-old CD73-/- and WT mice serum. We noticed some disturbances in the amino acid levels. CD73-/- mice were characterized by elevated concentration of - in particular-alanine, aspartic acid, glutamic acid, as well as lower concentration of tryptophan as compared to WT (Supplementary Table 1).
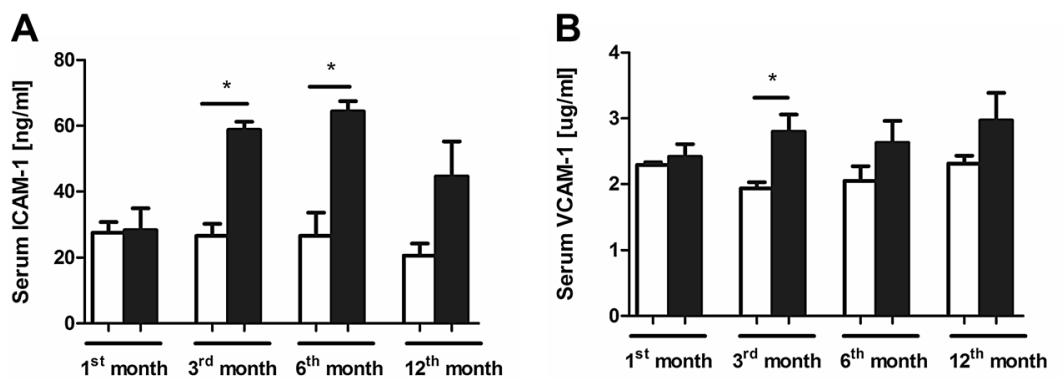

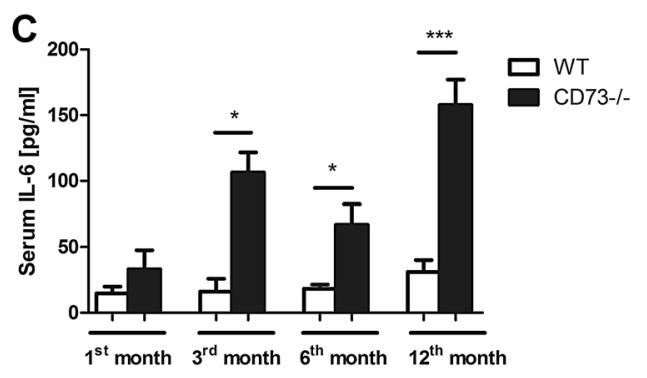

Fig. 1 Increase in IL-6 and adhesion molecules concentrations as a suggestion of an endothelium activation and pro-inflammatory phenotype of CD73-/- mice. Serum a ICAM-1; b VCAM-1 and c
IL-6 concentration in 1-, 3-, 6-, and 12- month-old CD73-/- and WT mice. All values are shown as mean \pm SEM ( $n=5$; Two-way ANOVA with post-hoc Tukey test and Student $t$ test: ${ }^{*} p$ 

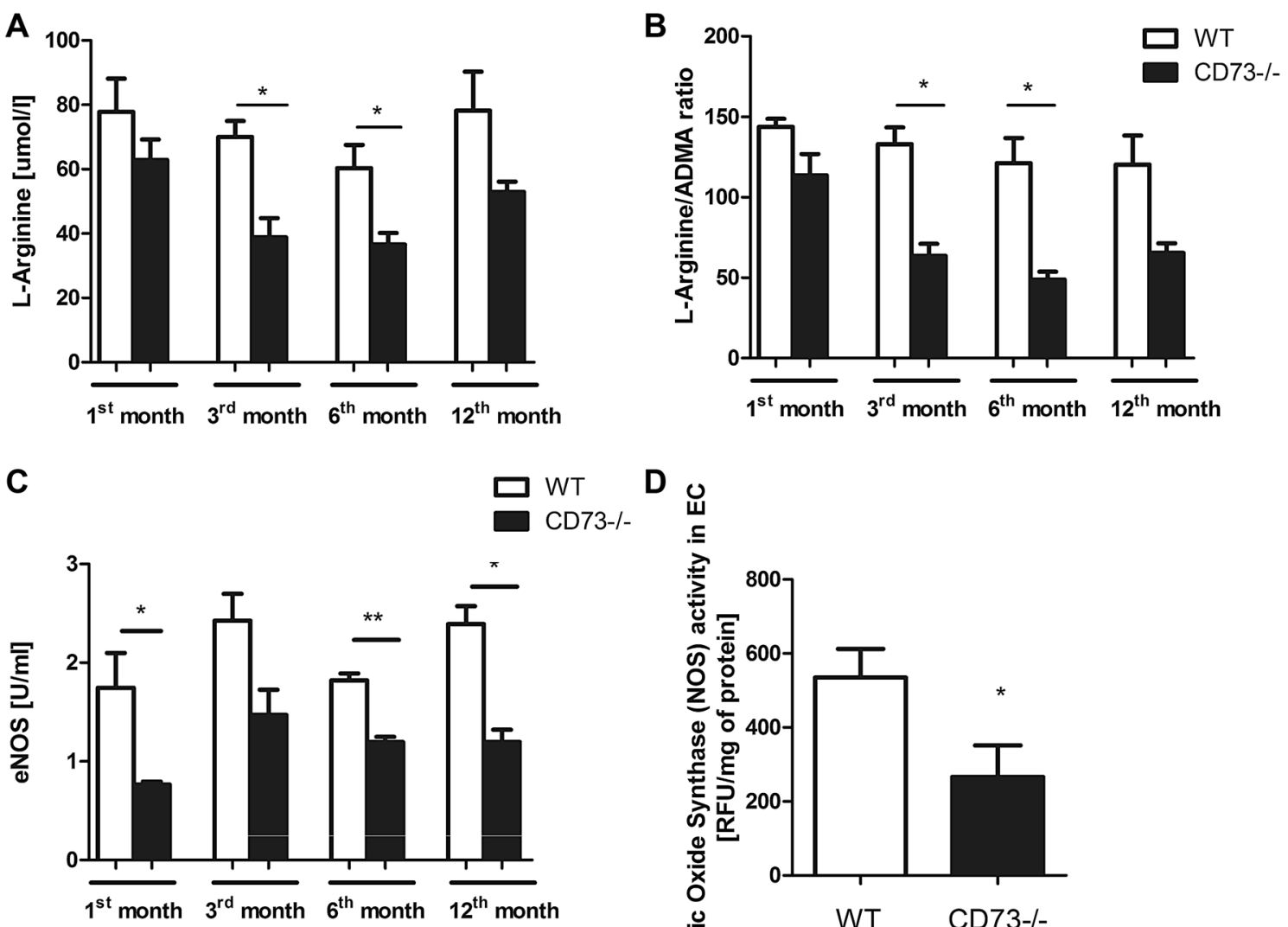

Fig. 2 Lower serum L-Arginine level and NOS activity in endothelial cells indicating endothelium dysfunction of CD73-/- mice. Serum a Arginine concentration, b Arginine/ADMA ratio, and c eNOS level of 1-, 3-, 6- and 12- month-old CD73-/- and WT mice. Values are shown as mean \pm SEM $(n=5$; Two-way ANOVA with post-hoc

Tukey test and Student $t$ test: $* p<0.05 ; * * p<0.01 ; * * p<0.001)$ d NOS activity in EC isolated from 2- month-old CD73-/- and WT mice lungs. Values are shown as mean \pm SEM $(n=3$-in each activity assay 5 wells for each group tested; Student t test: $* p<0.05$; ** $p$ $<0.01 ; * * * p<0.001)$
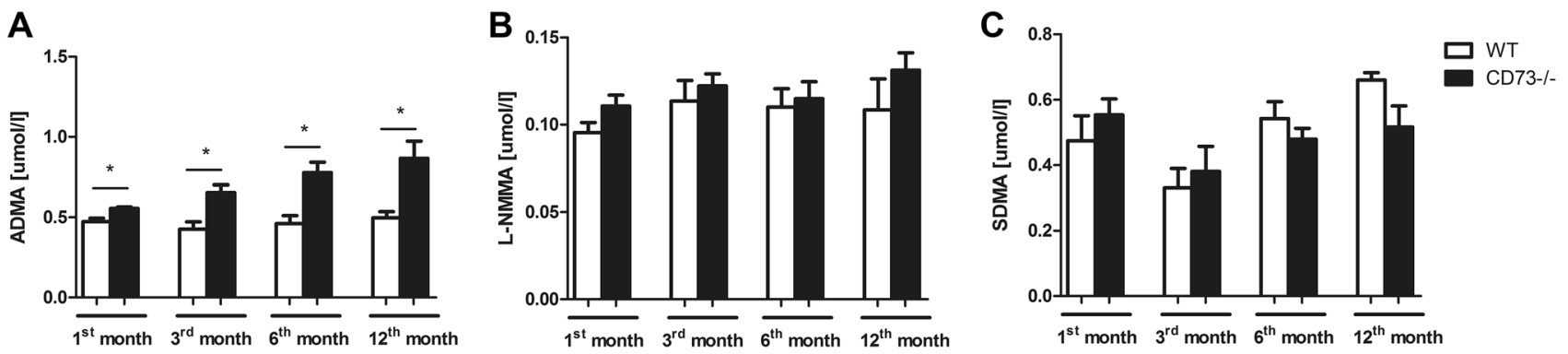

Fig. 3 The age-dependent ADMA increase in CD73-/- mice serum. Serum L-arginine analogs: a ADMA; b L-NMMA and $\mathbf{c}$ SDMA concentration of 1-, 3-, 6- and 12- month-old CD73-/- and WT mice.

\section{Discussion}

Our results support the concept of crucial role of the ecto5 '-nucleotidase activity in endothelium homeostasis. The lack of this activity in CD73-/- mice results in vascular
All values are shown as mean \pm SEM $(n=5$; Two-way ANOVA with post-hoc Tukey test and Student $t$ test: ${ }^{*} p<0.05$; ** $p<0.01$; *** $p<$ $0.001)$

endothelial dysfunction, proved by enhanced adhesion molecules and pro-inflammatory cytokine concentration. This study for the first time links the impaired L-Arginine metabolism with endothelial damage in CD73-deficient mice. Changes in the analyzed parameters were progressing with age of animals. 

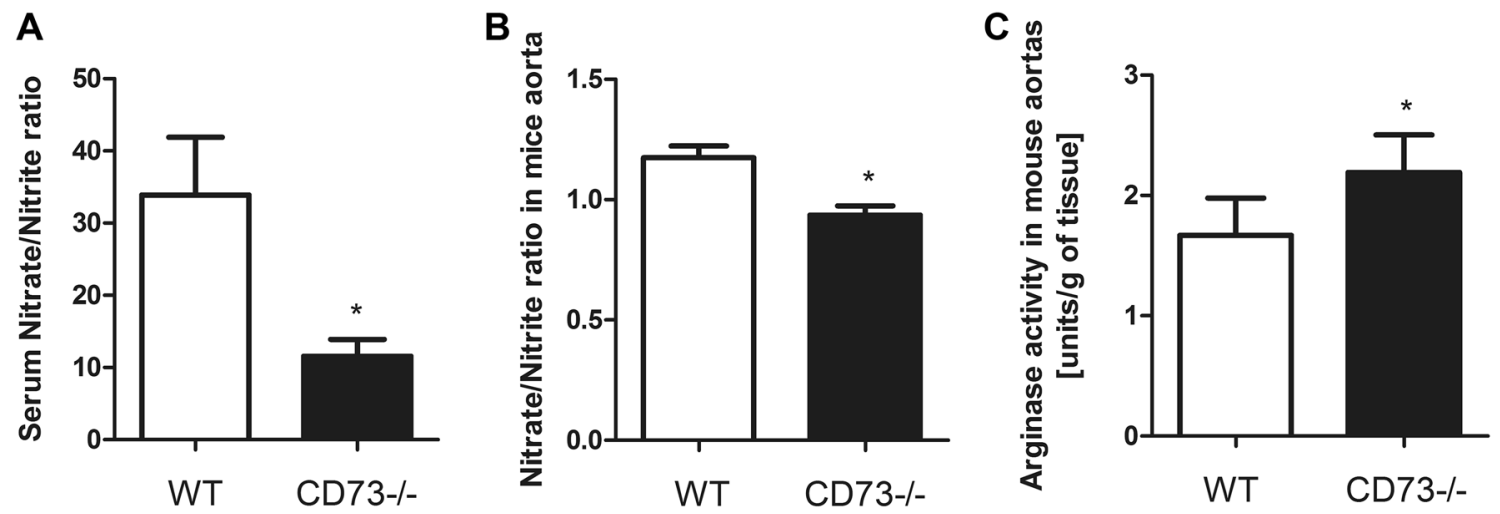

Fig. 4 Impaired nitric oxide metabolism in CD73-/- mice, indicated by the reduced serum and aortic nitrate/nitrite ratio. a Serum and $\mathbf{b}$ aortic nitrate/nitrite ratio, as well as $\mathbf{c}$ aortic arginase activity of 6 - month - old CD73-/- and WT mice. All values are shown as mean \pm SEM $(n=7$; Two-way ANOVA with post-hoc Tukey test and Student $t$ test: $* p<0.05 ; * * p<0.01 ; * * * p<0.001)$
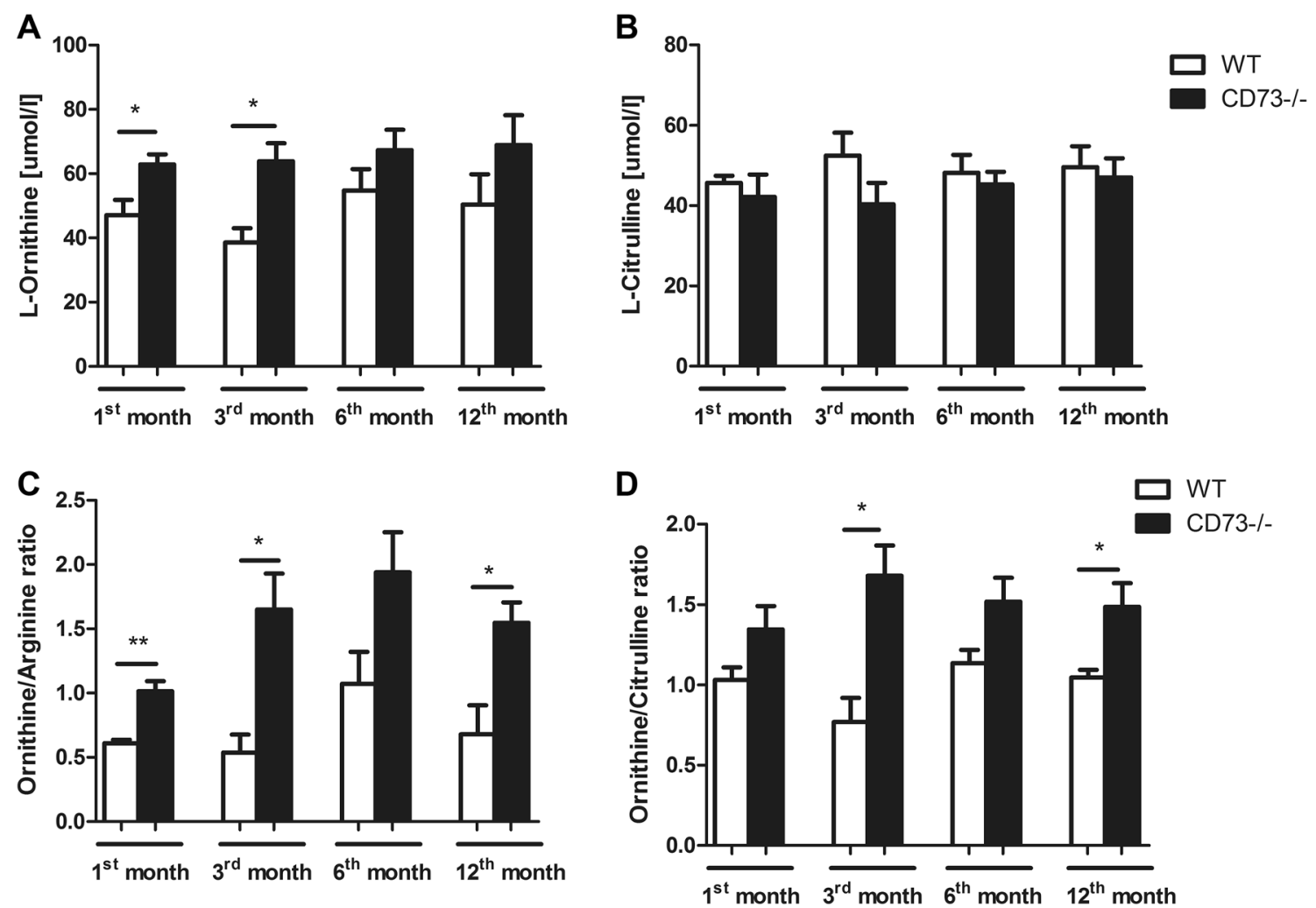

Fig. 5 Increased L-Ornithine/ L-Arginine and L-Ornithine/L-Citruline ratio in CD73-/- mice serum. a Ornithine and b Citruline, as well as c Ornithine/Arginine and d Ornithine/Citrulline ratios in 1-, 3-,

6-, and 12- month - old CD73-/- and WT mice serum. All values are shown as mean \pm SEM $(n=5$; Two-way ANOVA with post-hoc Tukey test and Student $t$ test: $* p<0.05 ; * * p<0.01$; *** $p<0.001$ )

The CD73 gene deletion resulted in significant increase in both adhesion molecules associated with endothelium activation-intracellular adhesion molecule 1 (ICAM-1) and vascular adhesion molecule 1 (VCAM-1), as well as considerably elevated concentration of the pro-inflammatory cytokines-interleukin 6 (IL-6) (Fig. 1) and tumor necrosis factor-alpha (TNF-alpha) (Fig. S2). This pro-inflammatory phenotype of CD73-/- mice was intensified with their age (Fig. 1). CD73 is highly expressed on vascular endothelial cells. Usually, endothelial cells (EC) are resistant to leukocyte adhesion. However, an active inflammatory process can stimulate adhesion molecules-ICAM-1 and VCAM-1 expression by EC, which results in the binding of leukocytes to the vessel wall and their transmigration into peripheral 

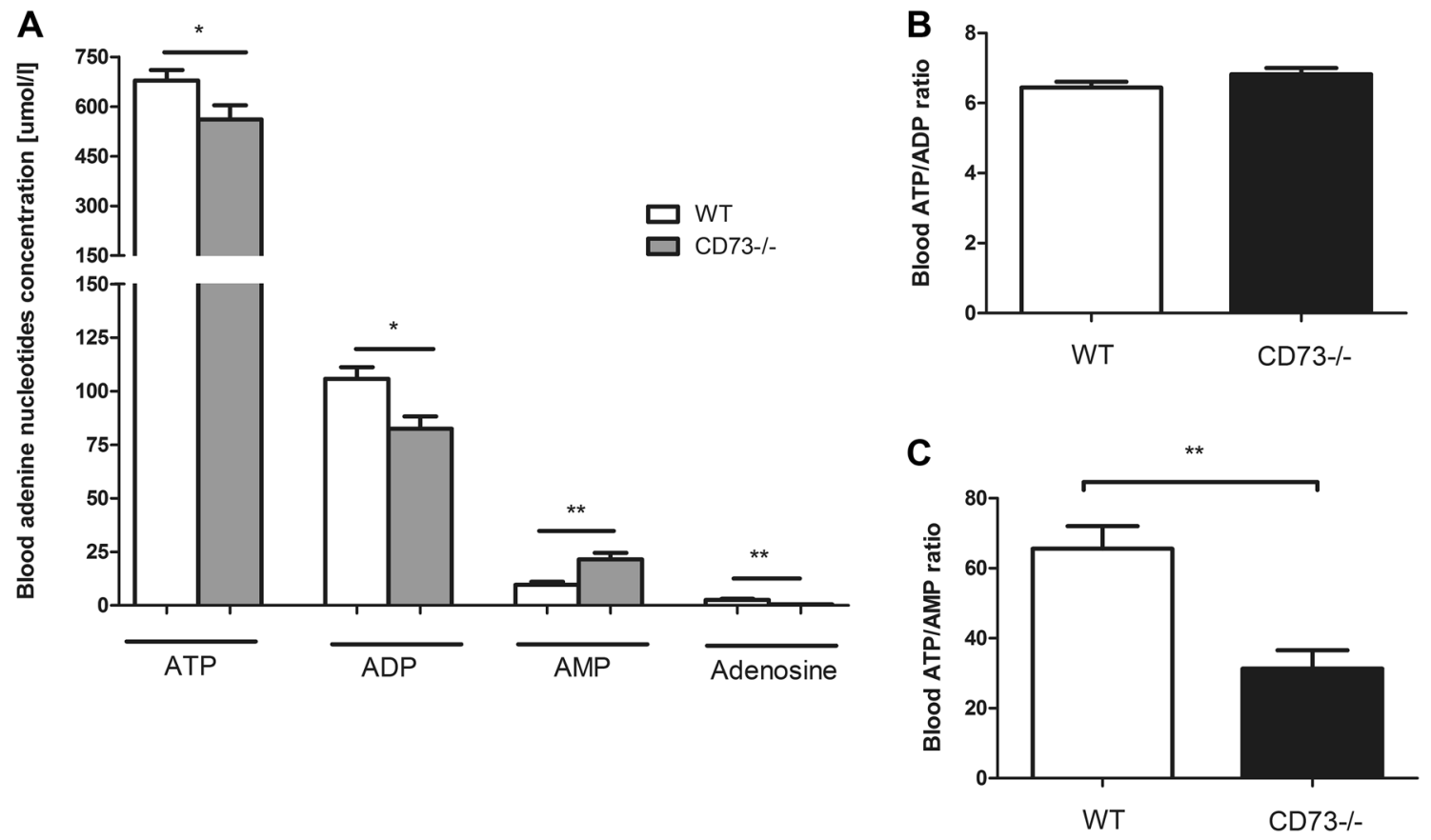

Fig. 6 Impaired adenine nucleotides pattern in CD73-deficient mice. a Blood adenine nucleotides and adenosine level, b blood ATP/ADP ratio and $\mathbf{c}$ blood ATP/AMP ratio in 6-month-old CD73-/- and WT

tissue [16]. Binding of leukocytes to EC results in an inhibition of CD73 activity [17]. Other studies also indicated that the monocytes adhesion in ex vivo-perfused carotid arteries of CD73-/- mice has been significantly increased and this pro-inflammatory effect was mediated by the VCAM-1 upregulation [18]. Colgan et al. demonstrated that CD73 deficiency had effect on the endothelial permeability. They observed exacerbation of hypoxia-induced vascular leak in various organs, such as lung, heart, or kidneys, in response to the lack of CD73 activity. Furthermore, a perivascular interstitial edema with inflammatory infiltrates surrounding the larger vessels of the pulmonary vasculature was noticed in CD73-/- mice subjected to hypoxia. This enhanced endothelial permeability may be related to the effect of the $\mathrm{A} 2 \mathrm{~B}$ receptor [19]. Activation of the A2A and A2B adenosine receptors on neutrophil surface contributes to an antiadhesive signal and thus decreases binding of neutrophils to endothelial cells. In addition, adenosine significantly diminishes release of the cytokines from the vasculature and leukocytes and therefore inhibits immune response and leukocyte extravasation [20]. All of these findings suggest that CD73 fulfills an important endothelial barrier function. Moreover, in vitro coculture experiments with EC and T lymphocytes demonstrated that genetic deletion of CD73 promotes the transendothelial migration of $\mathrm{T}$ lymphocytes and increases the expression of TNF-alpha, VCAM-1, and IFN-Y [21]. In addition, Burghoff et al. demonstrated that CD73-/- mice were characterized by significantly less mice blood. All values are shown as mean \pm SEM $(n=5$; Student $t$ test: $* p<0.05 ; * * p<0.01 ; * * * p<0.001)$

weight gain and a lower content of white adipose tissue, as well as increased free fatty acids (FFAs) and triglycerides in the serum. High levels of FFAs lead to the reactive oxygen species generation, as well as to the activation of TNF-alpha and IL-6 via the IKK/NF-KB inflammatory signaling and thus contribute to the endothelium cells damage [22, 23].

One of the possible consequences of endothelial dysfunction is the impairment in L-arginine metabolism and therefore decreased vascular nitric oxide (NO) activity. In the EC, $\mathrm{NO}$ is produced from L-arginine via the endothelial nitric oxide synthase (eNOS). NO is a key regulator of vasodilation and endothelium homeostasis. Moreover, it plays an important role in regulation of macrophage-induced cytotoxicity and platelet aggregation [24]. Since L-arginine is the only substrate for the eNOS-catalyzed reaction, its availability is criticial in controlling NO production [25, 26]. We observed considerably decreased L-arginine (Fig. 2a) and eNOS serum levels in all age groups of CD73-/- mice (Fig. 2c). Moreover, we found markedly lower NOS activity in EC isolated from CD73-/- mice lungs as compared to EC isolated from WT mice lungs (Fig. 2d), which was accompanied by reduced serum, but also aortic nitrate/nitrite ratio in CD73-/- group in comparison to control (Fig. 4a, b). Impaired production of NO leads to vasoconstriction, leukocyte adhesion and oxidative stress. Oxidative stress itself can also disrupt the production and activity of NO via its inactivation by free radicals and reduction of the tetrahydrobiopterin availability, which is a cofactor required for 
NO synthesis [27]. Our earlier data indicated significantly lowered plasma total antioxidant status in CD73-/- mice, which may suggest an increase production of free radicals [7], since oxygen radicals are scavenged by CD73-derived adenosine [28]. In addition, Saura M. et al found the association between increased IL- 6 concentration and reduction of the eNOS expression in endothelial cells via activation of the signal transducer and transactivator-2 (Stat-3) [29]. It has been also demonstrated that IL-6 may increase the binding between eNOS and caveolin-1, a caveolae scaffold protein that inhibits eNOS [30]. Furthermore, in addition to decreased serum L-arginine concentration, we noticed an elevated asymmetric dimethylarginine (ADMA) serum level (Fig. 3a) in all age groups of CD73-/- mice, what resulted in age-dependent L-Arginine/ADMA ratio reduction (Fig. 2b). Dimethylarginines-ADMA, NG-mono-methyll-arginine (L-NMMA) and NG-N'G-dimethyl-1-arginine (SDMA) are the products of the methylated proteins degradation. S-adenosylmethionine is a donor of the methyl groups, changing to S-adenosylhomocysteine (SAH) [31]. The formation of dimethylarginines is catalyzed by protein arginine methyltransferase type 1 and 2 (PRMT1, PRMT2). PRMT-1 is responsible for the LNMMA and ADMA formation, while PRMT-2 methylates proteins to release SDMA and L-NMMA. ADMA is an endogenous inhibitor of endothelial nitric oxide synthase (eNOS) that competes with its substrate L-arginine, impairing nitric oxide (NO) production and leading to endothelial dysfunction [32, 33]. Plasma ADMA has been found to be increased in patients with vascular pathologies [34]. Boger et al. found that ADMA concentration was better correlated with endothelial dysfunction than LDL cholesterol in hypercholesterolemic individuals and this increase in ADMA concentration was reversed by L-arginine administration [35]. Considering these data, our results are highly suggestive of age-dependent endothelial dysfunction in CD73-/- mice, leading to L-arginine metabolism disturbances.

We didn't find significant differences in other L-Arginine analogs-L-NMMA and SDMA-serum levels between CD73-/- and WT mice (Fig. 3). In addition to ADMA, L-arginine can also be methylated to produce SDMA and L-NMMA. ADMA and L-NMMA are competitive inhibitors of eNOS but SDMA does not appear to directly affect eNOS activity. However, all three methylarginines are able to interfere with L-arginine transport to EC, what results in cellular L-arginine deficiency [36]. L-NMMA is as potent as ADMA in eNOS inhibition, but its plasma concentration is approximately 10 times lower. Thus, ADMA seems to be a key player in eNOS activity reduction [32]. On the other hand, SDMA is considered to have a role in renal pathologies. The work of Zoccali et al. described the evaluation of ADMA concentration at the clinical outset of acute inflammation. Acute inflammation and accompanying nitrosative stress was characterized by an increase in the plasma level of ADMA and this phenomenon is accentuated in patients with relatively lower blood pressure. In this study, in contrast to ADMA, SDMA remained unchanged and was related to serum creatinine level [37]. Our results are consistent with these data. All age groups of CD73-/- mice were characterized by elevated serum L-ornithine concentration in comparison to control. There was no significant difference in serum L-citrulline level between studied groups. However, we found significantly increased ratios of ornithine/arginine and ornithine/citrulline in all CD73-/- age groups in comparison to controls (Fig. 5). Kövamees et al. also demonstrated similar observations in patients with type 2 diabetes, which is one of the pro-inflammatory diseases, and macrovascular complications [38]. These observations are consistent with the enhanced activity of arginase (Fig. 4c), responsible for the hydrolysis of L-arginine to urea and L-ornithine, in relation to NOS. Such changes in enzymatic activities observed in CD73 mutant mice may be also related to decreased NO production and thus, vascular dysfunction [39].

Considering the lack of the CD73 activity on the erythrocytes (data not shown), the significantly lower AMP hydrolysis rate observed in the blood of CD73-/- mice compared to WT (Figure S2) was most likely resulted from the CD73 deletion on circulating cells such as lymphocytes. However, it is well known that intracellular nucleotide concentrations are on milimolar level and extracellular concentrations are much lower (nmolar- $\mu$ molar) [40, 41]. Therefore, the observed changes in blood adenine nucleotide concentrations were caused by intracellular processes. Metabolic conditions such as oxidative stress or inflammation could lead to an increase requirements for ATP or reduce its regeneration, thus decreasing the overall ATP bioavailability [42]. Despite the decrease in ATP and ADP concentrations as well as a reduced ATP/AMP ratio in CD73-/- mice blood due to the enhanced ATP degradation, no disturbed values of adenylate energy charge in CD73-deficient animals were noticed $(0.93 \pm 0.02$ for WT; $0.90 \pm 0.01$ for CD73-/-). Moreover, ATP released from erythrocytes is an important regulator of synthesis by endothelial cells [43].

In conclusion, our study shows that the CD73 deletion leads to the development of age-dependent endothelial dysfunction in mice. Vascular damage is related to severe distrubances in L-arginine metabolism. The increase in CD73 activity could be protective in vascular pathologies.

Funding This study was supported by National Science Centre of Poland (2015/19/N/NZ1/03435) and the Polish Ministry of Science and Higher Education (01-0342/08/256). 


\section{Compliance with ethical standards}

Conflict of interest The authors declare no conflict of interest.

Open Access This article is distributed under the terms of the Creative Commons Attribution 4.0 International License (http://creativeco mmons.org/licenses/by/4.0/), which permits unrestricted use, distribution, and reproduction in any medium, provided you give appropriate credit to the original author(s) and the source, provide a link to the Creative Commons license, and indicate if changes were made.

\section{References}

1. Yegutkin GG (2008) Nucleotide- and nucleoside-converting ectoenzymes: important modulators of purinergic signalling cascade. Biochim Biophys Acta 1783(5):673-694

2. Antonioli L et al (2013) CD39 and CD73 in immunity and inflammation. Trends Mol Med 19(6):355-367

3. Thompson LF et al (2004) Crucial role for ecto-5'-nucleotidase (CD73) in vascular leakage during hypoxia. J Exp Med 200(11):1395-1405

4. Kumar V, Sharma A (2009) Adenosine: an endogenous modulator of innate immune system with therapeutic potential. Eur J Pharmacol 616(1-3):7-15

5. Esper RJ et al (2006) Endothelial dysfunction: a comprehensive appraisal. Cardiovasc Diabetol 5:4

6. d'Uscio LV et al (2014) Mechanisms of vascular dysfunction in mice with endothelium-specific deletion of the PPAR- $\delta$ gene. Am J Physiol Heart Circ Physiol 306(7):H1001-H1010

7. Zukowska P et al (2017) (2017) Deletion of CD73 in mice leads to aortic valve dysfunction. Biochim Biophys Acta 1863(6):1464-1472

8. Jalkanen S, Salmi M (2008) VAP-1 and CD73, endothelial cell surface enzymes in leukocyte extravasation. Arterioscler Thromb Vasc Biol 28(1):18-26

9. Ohta $\mathrm{M}$ et al (2013) Ecto-5'-nucleotidase, CD73, is an endothelium-derived hyperpolarizing factor synthase. Arterioscler Thromb Vasc Biol 33(3):629-636

10. Blume $\mathrm{C}$ et al (2012) Autoimmunity in CD73/Ecto-5' -nucleotidase deficient mice induces renal injury. PLoS ONE 7(5):e37100

11. Koszalka P et al (2004) Targeted disruption of cd73/ecto-5'-nucleotidase alters thromboregulation and augments vascular inflammatory response. Circ Res 95(8):814-821

12. Olkowicz M et al (2017) Application of a new procedure for liquid chromatography/mass spectrometry profiling of plasma amino acid-related metabolites and untargeted shotgun proteomics to identify mechanisms and biomarkers of calcific aortic stenosis. J Chromatogr A 1517:66-78

13. Kiss A et al (2010) The role of nitric oxide, superoxide and peroxynitrite in the anti-arrhythmic effects of preconditioning and peroxynitrite infusion in anaesthetized dogs. Br J Pharmacol 160(5):1263-1272

14. Groß F et al (2017) Copper amine oxidase 8 regulates argininedependent nitric oxide production in Arabidopsis thaliana. J Exp Bot 68(9):2149-2162

15. Smolenski RT et al (1990) Determination of sixteen nucleotides, nucleosides and bases using high-performance liquid chromatography and its application to the study of purine metabolism in hearts for transplantation. J Chromatogr 527(2):414-420

16. Frank PG, Lisanti MP (2008) ICAM-1: role in inflammation and in the regulation of vascular permeability. Am J Physiol Heart Circ Physiol 295(3):H926-H927
17. Henttinen T et al (2003) Adherent leukocytes prevent adenosine formation and impair endothelial barrier function by Ecto5'-nucleotidase/CD73-dependent mechanism. J Biol Chem 278(27):24888-24895

18. Zernecke A et al (2006) CD73/ecto-5'-nucleotidase protects against vascular inflammation and neointima formation. Circulation 113(17):2120-2127

19. Colgan SP et al (2006) Physiological roles for ecto-5'-nucleotidase (CD73). Purinergic Signal 2(2):351-360

20. Deaglio $S$ et al (2007) Adenosine generation catalyzed by CD39 and CD73 expressed on regulatory $\mathrm{T}$ cells mediates immune suppression. J Exp Med 204(6):1257-1265

21. Hasegawa T et al (2008) Ecto-5' nucleotidase (CD73)-mediated adenosine generation and signaling in murine cardiac allograft vasculopathy. Circ Res 103(12):1410-1421

22. Burghoff $\mathrm{S}$ et al (2013) Deletion of CD73 promotes dyslipidemia and intramyocellular lipid accumulation in muscle of mice. Arch Physiol Biochem 119(2):39-51

23. Ghosh A et al (2017) Role of free fatty acids in endothelial dysfunction. J Biomed Sci 24(1):50

24. Kamada Y et al (2001) Vascular endothelial dysfunction resulting from $\mathrm{L}$-arginine deficiency in a patient with lysinuric protein intolerance. J Clin Invest 108(5):717-724

25. Jamwal S, Sharma S (2018) Vascular endothelium dysfunction: a conservative target in metabolic disorders. Inflamm Res 67(5):391-405

26. Rodrigues-Krause $\mathbf{J}$ et al (2018) Association of L-arginine supplementation with markers of endothelial function in patients with cardiovascular or metabolic disorders: a systematic review and meta-analysis. Nutrients 11(1):15

27. d'Uscio LV et al (2003) Long-term vitamin C treatment increases vascular tetrahydrobiopterin levels and nitric oxide synthase activity. Circ Res 92(1):88-95

28. Le Hir M, Kaissling B (1993) Distribution and regulation of renal ecto-5'-nucleotidase: implications for physiological functions of adenosine. Am J Physiol 264(3 Pt 2):F377-F387

29. Saura $M$ et al (2006) Stat3 mediates interleukin-6 [correction of interelukin-6] inhibition of human endothelial nitric-oxide synthase expression. J Biol Chem 281(40):30057-30062

30. Hung MJ et al (2010) Interleukin-6 inhibits endothelial nitric oxide synthase activation and increases endothelial nitric oxide synthase binding to stabilized caveolin-1 in human vascular endothelial cells. J Hypertens 28(5):940-951

31. Tain YL, Hsu CN (2017) Toxic dimethylarginines: asymmetric dimethylarginine (ADMA) and symmetric dimethylarginine (SDMA). Toxins (Basel) 9(3):92

32. Sibal L et al (2010) The role of asymmetric dimethylarginine (ADMA) in endothelial dysfunction and cardiovascular disease. Curr Cardiol Rev 6(2):82-90

33. Tsikas D et al (2018) Asymmetric dimethylarginine (ADMA), symmetric dimethylarginine (SDMA) and homoarginine (hArg): the ADMA SDMA and hArg paradoxes. Cardiovasc Diabetol 17(1):1

34. Cooke JP (2000) Does ADMA cause endothelial dysfunction? Arterioscler Thromb Vasc Biol 20(9):2032-2037

35. Böger RH et al (1998) Asymmetric dimethylarginine (ADMA): a novel risk factor for endothelial dysfunction: its role in hypercholesterolemia. Circulation 98(18):1842-1847

36. Böger RH, Bode-Böger SM (2000) Asymmetric dimethylarginine, derangements of the endothelial nitric oxide synthase pathway, and cardiovascular diseases. Semin Thromb Hemost 26(5):539-545

37. Zoccali $\mathrm{C}$ et al (2007) Asymmetric dimethyL-arginine (ADMA) response to inflammation in acute infections. Nephrol Dial Transplant 22(3):801-806 
38. Kövamees $\mathrm{O}$ et al (2016) Amino acid metabolism reflecting arginase activity is increased in patients with type 2 diabetes and associated with endothelial dysfunction. Diab Vasc Dis Res 13(5):354-360

39. El Assar M et al (2016) Asymmetric dimethylarginine (ADMA) elevation and arginase up-regulation contribute to endothelial dysfunction related to insulin resistance in rats and morbidly obese humans. J Physiol 594(11):3045-3060

40. Gorman MW et al (2007) Human plasma ATP concentration. Clin Chem 53(2):318-325

41. González-Alonso J et al (2002) Erythrocyte and the regulation of human skeletal muscle blood flow and oxygen delivery: role of circulating ATP. Circ Res 91(11):1046-1055
42. Gutowska I et al (2010) Fluoride as a pro-inflammatory factor and inhibitor of ATP bioavailability in differentiated human THP1 monocytic cells. Toxicol Lett 196(2):74-79

43. Sprague RS et al (1996) ATP: the red blood cell link to NO and local control of the pulmonary circulation. Am J Physiol 271(6 Pt 2):H2717-H2722

Publisher's Note Springer Nature remains neutral with regard to jurisdictional claims in published maps and institutional affiliations. 